\title{
EL MODELO DE PARTIDO CARTEL Y EL SISTEMA DE PARTIDOS DE BRASIL*
}

\author{
The Cartel Party Model and the Brazilian Party System
}

\author{
PEDRO FLORIANO RIBEIRO \\ Universidad Federal de São Carlos (UFSCar), Brasil
}

\begin{abstract}
RESUMEN
El artículo analiza el actual sistema de partidos en Brasil bajo la lente conceptual del partido cartel de Katz y Mair, valiéndose de algunos de sus indicadores, pero sin pretender hacer una comprobación sistemática y global de la tesis. Se exploran tres conjuntos de indicadores: a) los vínculos de los partidos con la sociedad y el Estado; b) la competencia y la colusión entre los partidos principales; c) la organización y la estructura decisoria interna. Las conclusiones discuten la rentabilidad del modelo de cartelización con el objetivo de comprehender los patrones y transformaciones del sistema político brasileño y señalan las dificultades empíricas e inconsistencias teóricas y conceptuales del modelo, planteando una reelaboración teórica que lo restrinja a su núcleo esencial.
\end{abstract}

Palabras clave: Brasil, partido cartel, partidos políticos, sistemas de partidos, organización.

\begin{abstract}
The article analyzes the current Brazilian party system under the conceptual lens of Katz and Mair's cartel party theory $(1995,2009)$, using some of its indicators, but without trying a systematic and comprehensive test of the thesis. Three sets of indicators are explored: $a)$ the party linkages with society and state; $b$ ) competition and collusion among the major parties; $c$ ) organization and internal decision-making structure. The conclusions discuss the efficacy of cartelization model to understand the patterns and changes of the Brazilian political system, and point out the empirical difficulties and the theoretical and conceptual inconsistencies of the model, proposing a theoretical reelaboration which restricts it to its essential core.
\end{abstract}

Key words: Brazil, cartel party, political parties, party systems, organization.

* $\quad$ Este trabajo es resultado de un proyecto de investigación aún en desarrollo, financiado por la FAPESP (Fundação de Amparo à Pesquisa do Estado de São Paulo) bajo el título "Estado, partidos políticos y sociedad en el Brasil contemporáneo". Un esbozo del artículo se presentó en el VI Congreso de la Asociación Portuguesa de Ciencia Política, en marzo de 2012. Agradezco los comentarios y sugerencias hechos por Simone Diniz y Paulo Peres en el evento y las observaciones de los revisores anónimos de la Revista de Ciencia Política. 


\section{INTRODUCCIÓN}

Contrariando los pronósticos más pesimistas, el sistema de partidos brasileño ha adquirido, en los últimos años, un grado significativo de estabilidad. Las bancadas en el Congreso son cohesionadas y disciplinadas, y las amenazas de ingobernabilidad no se han consumado; los partidos han sido actores fundamentales en el funcionamiento del presidencialismo de coalición, formando y apoyando los gobiernos. La votación de los principales partidos está cada vez más nacionalizada, la volatilidad electoral se está estabilizando, y las tasas de identificación partidista son estables, siendo que casi la mitad del electorado manifiesta su preferencia por algún partido. Además, desde 1994 se nota una dinámica bipolar a nivel nacional -los bloques liderados por el Partido dos Trabalhadores (PT) y por el Partido da Social Democracia Brasileira (PSDB)-. Aunque la estabilización haya ocurrido en detrimento de otros atributos constitutivos de una democracia sólida, como representatividad y accountability, los partidos actuales desempeñan con razonable eficacia las funciones procedimentales (gobernativas y decisorias) fundamentales para el sistema político ${ }^{1}$.

Frente a estos fenómenos se pone una cuestión: ¿podría ocurrir, en Brasil, un proceso de cartelización del sistema de partidos? Alcántara Sáez (2004: 20) planteó esta hipótesis para los sistemas de América Latina como resultado de la consolidación de la democracia en la región. Nicolau (2010). Por otro lado, especuló sobre esta posibilidad para el caso brasileño (sin realizar una prueba sistemática), subrayando la reducida intensidad ideológica de los partidos, la posibilidad de participación de todas las fuerzas relevantes en el gobierno federal y la considerable dependencia con relación a los recursos (y reglamentos) del Estado.

El presente artículo propone analizar el actual sistema de partidos de Brasil bajo la perspectiva del modelo de partido cartel de Katz y Mair (1995; 2009). No ignoramos que el modelo fue desarrollado a partir de la experiencia europea, en un contexto muy específico, y tras décadas de transformaciones de los partidos. Sin embargo, la literatura reciente, teniendo en cuenta la inconsistencia global del modelo (debatida más adelante), ha empleado el concepto de una manera desagregada, aprovechando algunos de sus indicadores como estrategia de aproximación, marco analítico o incluso como hipótesis de trabajo, también para el análisis de sistemas en nuevas democracias, como los casos de España (López Nieto, 2011), Argentina, Bulgaria, República Checa (Kopecký, Spirova y Scherlis, 2011) y otros países excomunistas (Heurtaux y Roger, 2008) -además de los Estados Unidos, con una realidad muy distinta de la europea (Aucante, 2008)-. Teniendo en cuenta estos límites, no pretendemos hacer una comprobación sistemática de la hipótesis de cartelización; valiéndonos de algunos de sus indicadores, consideramos el modelo solo como una lente conceptual (de las muchas posibles) por la que se puede analizar un sistema de partidos, privilegiando algunos factores en detrimento de otros. 
El artículo empieza con una sistematización conceptual de la tesis del partido cartel, discutiendo también las principales fragilidades del modelo y presentando, a continuación, los indicadores empíricos que se explorarán. Enseguida se realiza una discusión sobre las condiciones previas que podrían influenciar la emergencia del fenómeno en Brasil. En las tres secciones siguientes se presentan los datos sobre tres conjuntos de indicadores: a) los vínculos entre partidos, sociedad y Estado; b) las estructuras decisorias de los partidos; c) los patrones de competencia y cooperación entre las principales fuerzas políticas. Las consideraciones finales conectan los puntos anteriores, reflexionando no solo sobre el caso brasileño, sino también sobre las inconsistencias teóricas y conceptuales del modelo, que ponen de manifiesto la necesidad de una reelaboración teórica que lo restrinja a su núcleo esencial.

\section{EL MODELO EN DISCUSIÓN}

La tesis del partido cartel (Katz y Mair, 1995, 1996, 2009) constituye uno de los conceptos más influyentes desarrollados en las últimas décadas. Siguiendo el camino de una amplia literatura que ha trabajado con el modelo ${ }^{2}$, se puede definir un sistema cartelizado como aquel en el que los principales partidos, de gobierno y oposición, cooperan con alguna frecuencia (de manera velada o explícita) para asegurar su posición dominante y su acceso privilegiado a recursos estatales decisivos para la supervivencia de todos y que minimizan los costos de derrotas electorales. La dependencia de los recursos estatales altera la correlación interna de poder a favor de la "cara pública" del partido (ocupantes de cargos públicos), que pasa a dominar los órganos partidistas. Este liderazgo partidista, a su vez, busca reforzar su posición mediante estrategias de concentración del poder interno que perjudican las bases del partido. En una relación circular de causalidad, la mayor autonomía del liderazgo facilita la flexibilidad y pragmatismo necesarios para la actuación concertada con otros partidos, al mismo tiempo en que el refuerzo de la posición del partido en el sistema aumenta su dependencia con relación a los recursos estatales y la fuerza del liderazgo en el plano interno. El entrelazamiento con el Estado, sumado a factores estructurales, como los niveles reducidos de participación y movilización social, la disolución de los clivajes clasistas, los altos costos de hacer política en tiempos de comunicación de masas y la notable profesionalización de la actividad política (que eleva los costos de eventuales derrotas), conducen al alejamiento de la sociedad: aunque los partidos deseen mantener vínculos sociales, las condiciones estructurales y del sistema político hacen indispensables tales lazos.

Hay algunos factores previos que motivan la cartelización. La reducción de las distancias ideológicas entre los principales partidos es uno de los requisitos indispensables: un escenario de gran polarización es poco propicio para el desarrollo de estrategias de colusión. En este sentido, tradiciones políticas de acomodación y negociación y arreglos institucionales consociativos ofrecen condiciones más favorables a la cartelización.

2 Katz y Mair (1995, 1996, 2002, 2009), Koole (1996), MacIvor (1996), Kitschelt (2000), Yishai (2001), Blyth y Katz (2005), Detterbeck (2005), Bardi (ed.) (2006), Scarrow (2006), Aucante y Dézé (ed.) (2008), Kopecký, Spirova y Scherlis (2011). 
Sobre el cuadro institucional, debe señalarse que los sistemas electoral y de partidos afectan la visión de las élites políticas sobre los lazos establecidos entre partidos y electores: cuanto más grande sea la percepción de vulnerabilidad de los vínculos sociales, mayor será la propensión a un giro hacia el Estado (generando un círculo vicioso). Por otro lado, las instituciones vigentes afectan la capacidad (y la necesidad) que tienen los partidos para manejar las reglas con finalidades típicas de los carteles: aumentar su acceso a los recursos estatales y poner (o reforzar) barreras a la entrada y al desarrollo de nuevos competidores. En tercer lugar, también se debe considerar la permeabilidad de las instituciones burocráticas y estatales a la influencia de los partidos: si el patronazgo partidista tiene amplio espacio para desarrollarse, están dadas las condiciones para la formación de partidocracias y, asimismo, de carteles. Ya un poder judicial independiente y activo (sobre todo las cortes supremas) puede representar un freno al desarrollo de estrategias de cartelización, así como la actuación agresiva de los medios de comunicación, capaces de inducir el desgaste público y la pérdida de legitimad de las principales fuerzas políticas (lo que puede abrir camino para nuevos competidores y partidos antisistema) (Detterbeck, 2005; Katz y Mair, 2009).

Desde su elaboración inicial, el modelo ha sufrido una multiplicidad de críticas. En el plano sistémico, se ha señalado que los autores atribuyen tonalidades demasiado fuertes a las fronteras contemporáneas entre sociedad civil y Estado, considerando las décadas de marcado intervencionismo estatal después de la Segunda Guerra Mundial, la acción de los grupos de interés y otros actores en los procesos de toma de decisiones, y, en décadas más recientes, las asociaciones público-privadas y redes de elaboración y gestión de políticas públicas. Con fronteras cada vez más tenues, no tiene mucho sentido hablar dicotómicamente de un desplazamiento de vínculos partidistas de la sociedad para el Estado. Asimismo, fenómenos como clientelismo, patronazgo, cooperación interpartidaria y relaciones estrechas entre partidos y Estado no constituyen ninguna novedad en el debate del siglo 20 y su aparición en escenarios muy diversos no permite correlacionarlos con patrones de competencia o tipos organizativos específicos. En tercer lugar, los test empíricos del modelo no siempre han alcanzado a diferenciar las acciones concertadas entre los partidos con la finalidad de beneficiar la clase política como un todo de aquellas que buscan promover solo los intereses de las fuerzas políticas principales. De igual modo, estos tests no han sido eficaces en delinear patrones de competencia claros: hay muchas experiencias distintas en términos de volatilidad, fragmentación, entrada de nuevos competidores, etc., sea entre los casos positivos de cartelización, sea entre los negativos. Por fin, tampoco se ha notado una correlación clara entre financiamiento público y cartelización: ante la presión de la opinión pública y de los medios de comunicación, no siempre los actores principales alcanzan a reforzar sus posiciones frente a los competidores más pequeños o aumentar las barreras de entrada contra nuevos partidos. En algunas ocasiones, incluso, los recursos públicos han ayudado a mantener el sistema más abierto y competitivo, frenando la cartelización que podría resultar de un sistema basado solamente en las donaciones de grandes empresas privadas, que prefieren invertir en los partidos principales. ${ }^{3}$

Las críticas, así como los indicadores empíricos que se emplean a continuación, se basan en la literatura citada anteriormente. 
Si los hallazgos empíricos en la dimensión sistémica no autorizan el delineamiento de relaciones causales y tendencias claras, en el plano organizativo las dificultades no son más pequeñas. Detterbeck (2005) destaca que los datos apuntan hacia una amplia variación de modelos organizativos entre los partidos contemporáneos, tanto en casos positivos como negativos de cartelización del sistema. Por otro lado, Yishai (2001) señala que partidos caracterizados individualmente como del tipo cartel pueden mantener y renovar sus vínculos sociales, aunque bajo nuevas bases.

Las reflexiones anteriores ponen de relieve el principal problema: la ausencia de coherencia interna del modelo, ya sea en términos lógicos o empíricos. Si en las democracias contemporáneas hay algunas tendencias observables en el plano sistémico y algunos patrones identificables con relación a los modelos organizativos, la tesis del partido cartel falla al intentar articular las dos dimensiones. A pesar de constituir, para sus creadores, componentes inseparables del modelo (Katz y Mair, 2009: 757, 763, nota 4), tales conexiones entre los planos sistémico y organizativo nunca han sido solucionadas de modo razonable en la literatura. Como señala Koole (1996), esta fragilidad podría incluso conducir a una contradicción no solo semántica, sino también lógica y conceptual insoluble: a partir de las contribuciones de Kirchheimer y Duverger, se puede considerar la existencia de un único partido catch all o un solo partido de masas en determinado sistema; con todo, ¿cómo hablar de un único partido cartel? Este punto se reanuda en las consideraciones finales.

\section{CONDICIONES PREVIAS}

En la historia política de Brasil, la fuerte dependencia de los partidos con relación a los aparatos del Estado ha sido siempre la tónica. A diferencia de Europa, donde muchos de los principales partidos se apoyaron, al menos en sus primeras décadas, en una sociedad bien organizada, en Brasil (como en gran parte de América Latina) las principales fuerzas políticas siempre estuvieron en la órbita estatal -desde la fundación, en el interior de aparatos ejecutivos y legislativos (la mayoría), o a partir del momento en que lograron posiciones de poder en distintas esferas (como es el caso del PT). En la raíz de esta simbiosis estatal se encuentran las peculiaridades del desarrollo histórico brasileño: una organización burocrática y estatal centralizada se constituyó antes de la sociedad y los partidos, que se articularon alrededor del aparato del Estado. Con una sociedad civil débilmente articulada, el clientelismo estatal despunta como una constante histórica: con escasas excepciones, el acceso a recursos institucionales y financieros vitales a la supervivencia de los partidos ha ocurrido primordialmente en espacios estatales, y no en las esferas sociales (Souza, 1983).

El cuadro institucional construido a partir de la redemocratización no ha cambiado esta situación. La legislación implantada en 1995 (Ley n 9096), que concedió autonomía a los partidos para la regulación de su organización interna, también elevó de manera exponencial la base estatal de financiamiento de las organizaciones (como se verá más adelante). El Estado brasileño además costea y regula el acceso de los partidos a los 
medios de comunicación, comprando espacios en la programación de radio y televisión no solo para las campañas electorales, sino también para las propagandas partidistas no electorales. La distribución de todos estos recursos es, en gran medida, proporcional al tamaño de los partidos en la Cámara de Diputados. El Estado también ofrece amplios espacios en la burocracia para la manutención y formación de militantes y apoyadores de los partidos y para la obtención de otros recursos organizativos importantes mediante mecanismos de patronazgo. El gobierno federal dispone de aproximadamente $21 \mathrm{mil}$ cargos de libre nombramiento por todo el país, siendo que el 14\% estaban ocupados por afiliados de los partidos en 2010 (Praça, Freitas y Hoepers, 2012). Datos recientemente recogidos registran 105 mil posiciones de libre nombramiento en los 27 gobiernos estaduales y alrededor de 500 mil en los ayuntamientos locales ${ }^{4}$. La distribución de esos cargos es la principal recompensa que los partidos y sus bancadas reciben por su apoyo legislativo al alcalde, gobernador o presidente.

La percepción de las élites partidistas sobre la fuerza de los vínculos sociales y sobre la necesidad de los apoyos estatales es otra dimensión que se debe considerar (Katz y Mair, 2009). Con relación a este punto, cabe notar que las élites políticas brasileñas tienen clara percepción sobre la necesidad del apoyo estatal para la viabilidad de la competencia democrática: los costos de campaña son extremamente elevados, no hay financiamiento público directo para las campañas y está prohibida la compra directa, por partidos y candidatos, de espacios para propaganda en radio y televisión. Además, la fragmentación del sistema de partidos, con un escenario altamente competitivo (especialmente en los principales estados del país), las tasas de volatilidad electoral aún significativas y la escasa legitimidad pública de los partidos y de la clase política (lo que quedó claro en las protestas masivas de junio de 2013) aumentan la percepción de las élites sobre la fragilidad de los vínculos con el electorado, incentivando acciones que buscan garantizar, en el firme suelo estatal, el acceso de los partidos a recursos organizativos vitales.

La concepción consociativa del sistema político brasileño (Santos, 2000) es otro factor que puede favorecer la cartelización. Con marcadas tendencias a la acomodación y negociación, los extremismos políticos se han vuelto residuales entre la clase política y el electorado brasileño. Hasta 2002, la clara postura oposicionista del PT y de sus tradicionales aliados de izquierda -Partido Comunista do Brasil (PC do B), Partido Democrático Trabalhista (PDT) y Partido Socialista Brasileño (PSB)-, y la retórica izquierdista de Lula da Silva y su partido, dificultaron la aproximación, en términos de valores y actitudes de sus élites, con los partidos más importantes de centro y derecha -PSDB, Democratas (DEM) y Partido do Movimento Democrático Brasileño (PMDB), que dominaron los bloques gubernamentales desde la redemocratización. La llegada del PT al gobierno federal en 2003 aceleró, por un lado, el proceso de revisión ideológica de la izquierda brasileña. Por otro, envolvió la construcción de alianzas duraderas con

4 Datos estaduales y locales recogidos por el Instituto Brasileño de Geografía y Estadística (IBGE) y presentados en: "Governadores controlam máquina de 105 mil cargos sem concurso público". Jornal O Estado de São Paulo, 31 de marzo de 2013. "Prefeituras do país criam 64 mil cargos para nomeação política em quatro anos". Jornal O Estado de São Paulo, 14 de julio de 2013. 
algunos de los mismos partidos que habían integrado la base de gobierno de Fernando Henrique Cardoso (como el PMDB, el Partido Trabalhista Brasileiro [PTB], el Partido Progresista [PP] y el Partido Liberal/Partido de la República [PL/PR]). ${ }^{5}$ Estos factores, además de la posición moderada de la mayoría del electorado, contribuyeron para reducir la distancia ideológica entre los miembros del Congreso Nacional, mensurada a partir de sus valores y opiniones (Power y Zucco, 2011). Para Lucas y Samuels (2011), la distinción de corto plazo entre el gobierno y la oposición se ha vuelto más relevante que la separación de derecha e izquierda. En un escenario poco polarizado, hay más posibilidades de colusión entre los actores políticos.

Por fin, tras un período de transición del bipartidismo para el multipartidismo en la década de los 80 y después de un período de reacomodación de la clase política entre 1990 y 1994, el sistema de partidos de Brasil adquirió un grado de estabilidad inédito para los patrones del país -al menos hasta 2011-. De los principales partidos, el más nuevo era, hasta recientemente, el PSDB, fundado en 1988. Una dinámica bipolar ha sido la tónica en la política brasileña desde 1994, con dos bloques alternándose en el comando del país: uno de centro-izquierda, liderado por el PT, y otro de centro-derecha, liderado por el PSDB. En el centro (PMDB) y en la derecha (PTB, PR, PP) del espectro político hay un conjunto de partidos de perfil "fisiológico" dispuestos a participar en cualquiera de los gobiernos. Las dos elecciones de Cardoso (1994 y 1998) y las tres victorias del PT (2002, 2006 y 2010), así como la dinámica en el Congreso en los períodos entre los comicios, se rigieron por tal estructuración bipolar, con espacios muy reducidos para terceras fuerzas. Como las elecciones para Presidencia, Congreso Nacional (Senado y Cámara), gobiernos estaduales y asambleas estaduales ocurren simultáneamente (desde 1994), la dinámica bipolar de la disputa presidencial poco a poco empezó a afectar los comicios en los estados, configurando un patrón de competencia más nítido -lo que puede estar generando, incluso, una reducción de las tasas de volatilidad electoral (Limongi y Cortez, 2010; Melo e Câmara, 2012)-.

Por otro lado, hay algunos factores que juegan en contra el proceso de cartelización. En primer lugar, las barreras de entrada en el sistema político brasileño, así como para el acceso a los recursos estatales, son bastante flojas. No hay una cláusula de desempeño uniforme para la entrada de los partidos en las esferas legislativas: gracias a esto y también a la posibilidad de hacerse coaliciones electorales para las disputas legislativas, hasta los pequeños partidos consiguen elegir representantes. El desempeño del partido en la votación para la Cámara determina su participación en la distribución de los recursos estatales más importantes: el fondo partidario (recursos del Tesoro Nacional directamente entregados a los directorios nacionales de los partidos) y el tiempo para propaganda en radio y televisión. Desde 2007, el 5\% del fondo partidario se divide igualmente entre todos los partidos y el 95\% se distribuye de acuerdo con la última votación para la Cámara. Para el tiempo de radio y TV en las campañas se reparte igualmente un tercio entre todos los partidos (o coaliciones) con candidatos y dos tercios se dividen en proporción al tamaño de las

5 En 2006, el PL cambió su nombre para PR. En 2007, el Partido da Frente Liberal (PFL), que enfrentaba una grave crisis en consecuencia de su salida del gobierno federal, alteró su nombre para Democratas (DEM). 
bancadas en la Cámara. De este modo, incluso los partidos insignificantes tienen acceso a estos recursos. El actual criterio de división del fondo partidario es menos favorable a los grandes partidos que las reglas anteriores, vigentes hasta 2006-07. La ley implantada en 1995 (Ley n ${ }^{\circ}$ 9096) determinaba que una cláusula de desempeño entraría en vigencia a partir de las elecciones generales de 2006: solamente los partidos que obtuviesen el $5 \%$ de los votos en la elección para la Cámara tendrían derecho a funcionamiento parlamentario y participarían en la división del $99 \%$ del fondo partidario y de la gran mayoría del tiempo de radio y TV (el restante de los recursos se dividiría igualmente entre todos). En 2006, siete partidos superaron la cláusula (PMDB, PT, PSDB, PFL/DEM, PP, PSB y PDT) y los partidos medianos y pequeños recurrieron a la Justicia. En consecuencia, el Supremo Tribunal Federal (STF) declaró que la cláusula era inconstitucional y entonces el actual criterio de distribución fue aprobado (se verán más detalles adelante).

La fragilidad de las barreras de entrada quedó evidente en 2011 con la creación del Partido Social Democrático (PSD) por el exalcalde de São Paulo, Gilberto Kassab. Atrayendo a parlamentarios de los partidos de centro y derecha (principalmente del DEM), muchos de ellos interesados en acercarse al gobierno federal, el partido ya nació con la cuarta bancada más grande en el Congreso. En junio de 2012, una decisión del Tribunal Superior Electoral (TSE) reforzó los planes de la agremiación: aun sin haber participado en las elecciones generales, el partido obtuvo el derecho de recibir cuotas del fondo partidario proporcionales a la votación total de los diputados que se habían cambiado para el PSD, después de elegidos en 2010 por otros partidos. Al demostrar que las puertas del sistema están abiertas a nuevos competidores, el éxito del PSD está motivando nuevos movimientos en el escenario político, como la creación de un partido ambientalista (La "Red Sustentabilidad") por la exministra del gobierno de Lula (y candidata a la Presidencia) Marina Silva.

El episodio ilustra con perfección la segunda barrera a la cartelización del sistema político brasileño: la actuación del poder judicial, especialmente del TSE y STF, órganos que han representado los espacios finales de apelación de los partidos medianos y pequeños contra los grandes. La actuación muchas veces agresiva de los grandes grupos de comunicación (con un noticiario político que privilegia los casos de corrupción) también constituye una barrera contra la adopción de estrategias explícitas de colusión. Por fin, si la desconfianza en los partidos está generalizada en América Latina, el caso brasileño se sitúa como uno de los más críticos. Las encuestas ubican los partidos en la última colocación del ranking de confianza del electorado brasileño en sus instituciones. En 2011, solo el 43\% de los electores consideraban imposible una democracia sin partidos. Entre los jóvenes la percepción es todavía más negativa, lo que se hizo evidente en las masivas manifestaciones callejeras de junio de 2013, que asustaron a la clase política: el 83\% de los manifestantes afirmaron que no se sentían representados por los políticos y el $89 \%$ no se veían representados por los partidos ${ }^{6}$. En tal escenario, la construcción de estrategias del tipo cartel no es tarea sencilla.

\footnotetext{
El ranking de confianza en las instituciones ubica los partidos en la última colocación desde 2009, entre 18 instituciones brasileñas, políticas o no. Este ranking y las encuestas con los manifestantes fueron realizadas por el IBOPE. Para los datos de 2011 sobre la democracia sin partidos: Latinobarómetro (2011).
} 


\section{PARTIDOS, ESTADO Y SOCIEDAD}

Algunos indicadores empíricos, así como los efectos esperados de determinadas instituciones, ya han sido explorados en los párrafos anteriores. En esta sección se analizan otros indicadores.

Los datos sobre el financiamiento directo e indirecto de los partidos brasileños confirman la dependencia de los recursos estatales. En 2012, el resarcimiento (mediante exención fiscal) a las empresas de radio y televisión para la difusión de propagandas de los partidos ultrapasó los 300 millones de dólares, llegando a casi 500 millones en 2010, año de elecciones generales (Gráfico 1). Con relación a los recursos directos, la ley de partidos implantada en 1995-96 multiplicó el fondo partidario. De 1996 a 2010 el importe total del fondo ha sido corregido según la cantidad de electores y la inflación. En 2011, una acción concertada entre todos los partidos (para pagar las deudas de campaña) aumentó el fondo en 50 millones de dólares, permaneciendo para los años siguientes. ${ }^{7}$ En 2012, los 30 partidos registrados recibieron 178 millones de dólares (Gráfico 1).

Gráfico 1: Fondo partidario y gastos con la comunicación de los partidos (exención fiscal), 1994-2012 (US\$ millones)

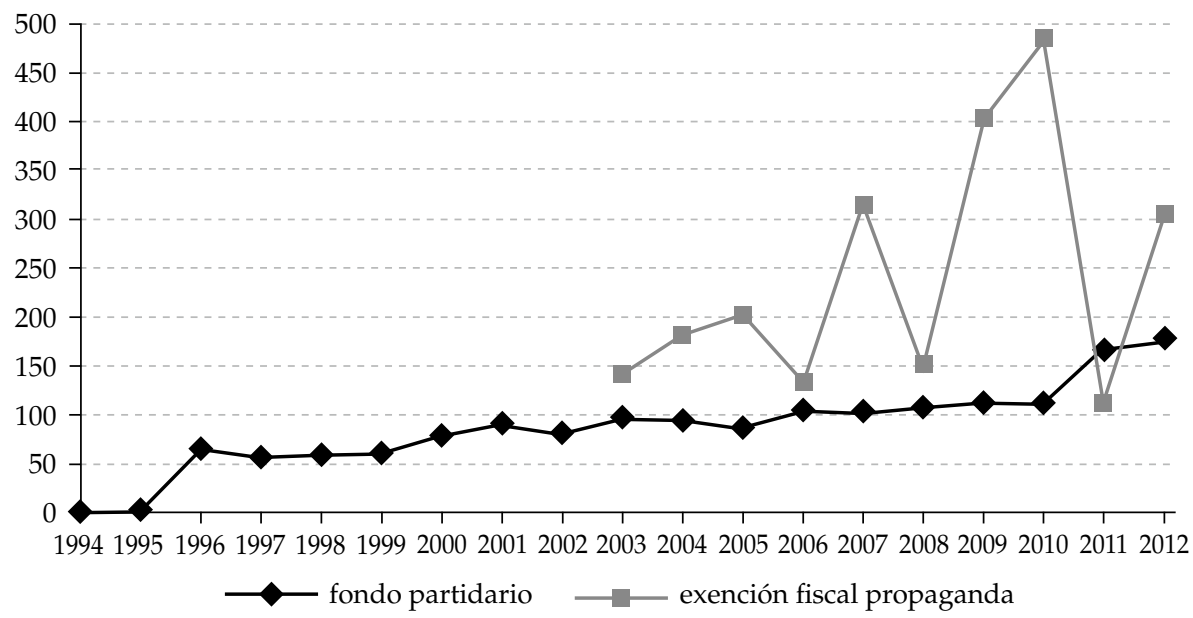

Fuente: datos brutos recogidos en el TSE (fondo partidario) y en la Receita Federal de Brasil (informes sobre el resarcimiento, mediante exención fiscal, de las empresas de radio y televisión para las emisiones de la propaganda institucional y electoral de los partidos). Los valores originales fueron actualizados para enero de 2013 según el INPC (Índice Nacional de Precios al Consumidor) y convertidos al dólar americano según el cambio de 31 de enero de 2013.

7 “Congresso 'estatiza' dívidas de campanha ao turbinar verba pública para partidos". Jornal O Estado de São Paulo, 14 de enero de 2011. 
La legislación permite a los partidos y candidatos la captación de recursos privados. Sin embargo, la subvención pública es, hoy, la fuente más regular y segura de financiamiento para la clase política. ${ }^{8}$ En años electorales (años pares), principalmente de elecciones generales, los grandes partidos logran captar abundantes sumas de recursos con las empresas privadas y, así, disminuyen la dependencia de los recursos estatales (Tabla 1). Solo el PT posee, de manera significativa, una fuente alternativa a los recursos del Estado y de las grandes compañías: la contribución obligatoria de los titulares de cargos públicos, elegidos o no (los recursos de los militantes "comunes" son insignificantes). Teniendo en cuenta todo el sistema de partidos, los recursos estatales directos significaron más del $80 \%$ del total de ingresos en los últimos años no electorales. Para los nueve partidos principales, el fondo partidario representó el 90\% del total de ingresos en 2007, 2009 y 2011; en los años electorales de 2008, 2010 y 2012 significó el 61,6\%, el 33,6\% y el 40,4\%, respectivamente (siempre los promedios). Considerando solo los cuatro principales (PT, PMDB, PSDB y DEM), que disponen de amplia capacidad de atracción de recursos, el peso del financiamiento público fue de $44 \%$ en 2008 y de un cuarto del total de ingresos en 2010 y $2012 .{ }^{9}$

Tabla 1: $\quad$ Peso del fondo partidario estatal en el financiamiento de los partidos, 2007$2012(\mathrm{en} \%)$

\begin{tabular}{lcccccc}
\hline & 2007 & 2008 & 2009 & 2010 & 2011 & 2012 \\
\hline PT & 59,6 & 27,3 & 61,1 & 13,3 & 46,6 & 16,6 \\
PMDB & 93,6 & 73,4 & 92,8 & 23,7 & 88,9 & 27,2 \\
PSDB & 84,4 & 37,5 & 87,5 & 18,7 & 93,1 & 26,7 \\
DEM & 98,3 & 36,6 & 94,7 & 42,1 & 99,1 & 36,1 \\
PP & 99,1 & 81,5 & 99,8 & 43,7 & 98,8 & 58,9 \\
PSB & 91,0 & 46,2 & 97,8 & 23,2 & 95,8 & 24,9 \\
PDT & sin datos & 92,1 & 94,4 & 54,4 & 97,1 & 56,1 \\
PR & 95,1 & 69,8 & 90,3 & 25,9 & 95,7 & 72,4 \\
PTB & 100,0 & 90,1 & 95,6 & 58,1 & 98,5 & 56,7 \\
PSD & - & - & - & - & 31,7 & 28,7 \\
Promedio ${ }^{1}$ & 90,1 & 61,6 & 90,5 & 33,6 & 90,4 & 40,4 \\
Promedio general: todos los partidos & 83,7 & 63,5 & 83,3 & 51,1 & 86,7 & 62,4 \\
\hline
\end{tabular}

Fuente: Declaraciones de ingresos y gastos de los directorios nacionales, entregados anualmente al TSE. ${ }^{1}$ El PSD ha sido incluido en el cálculo de los partidos más grandes solo en 2012.

8 Los partidos usan el fondo para el mantenimiento de sus institutos de investigación y de sus actividades cotidianas, estando permitido incluso transferir recursos para los directorios estaduales y locales e invertir el dinero en las campañas electorales.

9 Los datos se refieren solo a los ingresos de los directorios nacionales. Los órganos estaduales y locales no reciben recursos directamente del Estado, pero pueden captar sus propias donaciones privadas. Hasta mediados de los años 90, el PT no aceptaba donaciones de empresas. Para el proceso de profesionalización de las campañas (especialmente la presidencial) y de otras actividades del aparato, a partir del año 2000 el partido empezó a buscar estos recursos (Ribeiro, 2010). 
Por otro lado, los datos sobre membresía, identificación partidista y difusión territorial no delinean una tendencia clara de erosión de lazos entre partidos y electores en Brasil, como esperado según el modelo de Katz y Mair (2009). Los partidos han intentado, al menos en la última década, ampliar sus contingentes de afiliados: la membresía total a los partidos superaba los 15 millones de afiliados en 2012, casi el 11\% del electorado (Tabla 2). ${ }^{10}$ Sobre la identificación partidista cabe señalar la estabilidad de las tasas (Tabla 3): con valores agregados variando alrededor del $45 \%$, las oscilaciones no ocurren de manera brusca, pero sí se modifican a largos plazos, como consecuencia de estrategias más o menos exitosas en la construcción de imágenes sólidas -con el PT representando el caso más relevante-. Finalmente, la capilaridad organizativa de los partidos brasileños es bastante significativa. Principalmente el PT, el PMDB y el PSDB son capaces de llegar hasta los rincones más pequeños y alejados, lo que constituye una importante ventaja competitiva frente a otros actores políticos (Tabla 4).

\section{RELACIONES INTERNAS DE PODER}

La tradición de organización partidista en Brasil apunta hacia una notable descentralización federalizada, con las secciones locales y, principalmente, estaduales disfrutando de amplia autonomía decisoria. Los partidos del actual régimen, a excepción del PT, siguieron con esta forma de organización. Sin embargo, a partir de mediados de los años noventa, los

Tabla 2: Membresía de los partidos políticos, 2002-2012 (en millones de electores)

\begin{tabular}{lcccc}
\hline & 2002 & 2006 & 2010 & 2012 \\
\hline PMDB & 2,2 & 2,0 & 2,3 & 2,4 \\
PT & 0,8 & 1,1 & 1,4 & 1,6 \\
PSDB & 1,1 & 1,1 & 1,3 & 1,4 \\
PFL/DEM & 1,0 & 1,0 & 1,1 & 1,1 \\
PP & 1,4 & 1,3 & 1,4 & 1,4 \\
PDT & 1,0 & 1,0 & 1,1 & 1,2 \\
PTB & 0,8 & 1,0 & 1,2 & 1,2 \\
PL/PR & 0,5 & 0,7 & 0,7 & 0,8 \\
PSB & 0,4 & 0,3 & 0,5 & 0,6 \\
Otros partidos (agregados) & 2,0 & 2,2 & 2,9 & 3,4 \\
Total afiliados Brasil & 11,1 & 11,6 & 13,9 & 15,1 \\
Afiliación total/ electorado total & $9,7 \%$ & $9,2 \%$ & $10,2 \%$ & $10,8 \%$ \\
\hline
\end{tabular}

Fuente: datos oficiales del TSE.

10 Aunque los mecanismos de registros se hayan desarrollado en los últimos años, las cifras tienden a estar sobrestimadas, principalmente para los partidos más pequeños, incapaces de mantener registros precisos. 
Tabla 3: $\quad$ Tasas de identificación partidista, 1989-2012 (\%)

\begin{tabular}{cccccccc}
\hline & PT & PMDB & PSDB & PFL/DEM & $\begin{array}{c}\text { Otros } \\
\text { (agregados) }\end{array}$ & $\begin{array}{c}\text { Total id. } \\
\text { partidista }\end{array}$ & $\begin{array}{c}\text { Sin } \\
\text { preferencia }\end{array}$ \\
\hline 1989 & 10 & 15 & 1 & 5 & 13 & 44 & 56 \\
1992 & 14 & 18 & 2 & 5 & 14 & 53 & 47 \\
1994 & 15 & 17 & 4 & 4 & 8 & 48 & 52 \\
1996 & 12 & 15 & 5 & 6 & 8 & 46 & 54 \\
1998 & 12 & 14 & 5 & 5 & 7 & 43 & 57 \\
2000 & 15 & 12 & 3 & 6 & 9 & 45 & 55 \\
2002 & 18 & 9 & 4 & 6 & 6 & 42 & 58 \\
2004 & 22 & 8 & 5 & 4 & 6 & 45 & 53 \\
2006 & 17 & 7 & 6 & 3 & 6 & 39 & 61 \\
2008 & 21 & 8 & 5 & 2 & 7 & 43 & 57 \\
2010 & 23 & 6 & 6 & 1 & 11 & 46 & 54 \\
2012 & 27 & 5 & 4 & 1 & 9 & 46 & 54 \\
\hline
\end{tabular}

Fuente: Instituto Datafolha (promedios anuales de las encuestas del instituto).

Tabla 4: Difusión territorial de los principales partidos (2012)

\begin{tabular}{lccccccccc}
\hline & PT & PMDB & PSDB & PDT & PP & DEM & PSB & PR & PTB \\
\hline $\begin{array}{l}\text { Directorios } \\
\text { municipales (n) }\end{array}$ & 4443 & 3357 & 2589 & 1289 & 1068 & 1093 & 771 & 40 & 32 \\
\hline $\begin{array}{l}\text { Comisiones } \\
\begin{array}{l}\text { Provisionales } \\
\text { Municipales (n) }\end{array}\end{array}$ & 937 & 1571 & 2195 & 2899 & 3111 & 3265 & 3530 & 4388 & 3910 \\
\hline $\begin{array}{l}\text { Organización Total. } \\
\text { (direct. + comis.) (n) }\end{array}$ & 5380 & 4928 & 4784 & 4188 & 4179 & 4358 & 4301 & 4428 & 3942 \\
\hline $\begin{array}{l}\text { \% de organización } \\
\text { total }^{1}\end{array}$ & 96,7 & 88,6 & 86,0 & 75,3 & 75,1 & 78,3 & 77,3 & 79,6 & 70,8 \\
\hline
\end{tabular}

Fuente: datos del TSE de agosto de 2012, recogidos por el equipo del Centro de Estudos de Partidos Políticos - UFSCar. ${ }^{1}$ Cálculo en relación con el total de ciudades brasileñas (5565).

líderes nacionales de los principales partidos empezaron a promover reformas internas que están, en algunos casos, cambiando las estructuras de toma de decisiones.

En el PT, DEM, PMDB y PSDB, muchas de las prerrogativas de los directorios han sido transferidas para las comisiones ejecutivas, instancias reducidas y más ágiles. Los líderes del PSDB y DEM reformaron sus órganos principales (como la Ejecutiva Nacional), ampliando la participación de parlamentarios y otros "notables", en la condición de miembros natos, no 
elegidos en convenciones o congresos y crearon (así como el PMDB) nuevos órganos, como Consejos Nacionales o Políticos, en los que los criterios de antigüedad y prestigio también son los más importantes. ${ }^{11}$ De este modo, las decisiones más relevantes se encuentran, hoy, más concentradas en la cúpula dirigente. Los dos partidos también han reforzado la articulación orgánica entre la cúpula nacional y las secciones subnacionales, mitigando el carácter federalizado de las organizaciones. Los líderes han ampliado las posibilidades estatutarias de intervención jerárquica, principalmente de la cúpula nacional sobre las demás secciones, y estas intervenciones se han vuelto más comunes, especialmente por razones electorales -como prohibiciones de alianzas-. Con esas reformas, el PSDB y el DEM son, hoy, partidos más articulados y centralizados que el PMDB, cuya estructura sigue siendo la más descentralizada, incluso con criterios regionales de formación de los órganos nacionales (Ferreira, 2002; Ribeiro, 2013).

Además, respecto de la distribución interna de los recursos estatales, solamente el PMDB mantiene una cuantiosa descentralización del dinero del fondo partidario: en el PT, PSDB y DEM hay una tendencia a la concentración de estos ingresos en las cúpulas nacionales, en perjuicio de las secciones estaduales (Tabla 5). Asimismo, las élites de todos los principales partidos (a excepción del PT) también han recurrido, con una frecuencia cada vez mayor, a la disolución de directorios estaduales y locales como recurso de control interno y enforcement sobre estos niveles y también como estrategia en las disputas entre facciones, sustituyendo los órganos por comisiones provisorias indicadas por las cúpulas (Guarnieri, 2011).

Como ocurre en los demás partidos más importantes, en el PT, la Ejecutiva Nacional se ha transformado en la principal instancia de decisión, suplantando el Directorio Nacional y las propias convenciones, congresos y encuentros de delegados. Sobre el partido de Lula hay un consenso sobre la forma innovadora de su organización inicial, con canales de participación de las bases, control sobre los parlamentarios, articulación interna intensa, vínculos firmes con la sociedad y una acción política centralizada nacionalmente. En los últimos años, algunas de estas características han sido disipadas, o incluso han

Tabla 5: Proporción del fondo partidario transferido por los directorios nacionales a las secciones estaduales (\%)

\begin{tabular}{lccccccc}
\hline & 2007 & 2008 & 2009 & 2010 & 2011 & 2012 & promedio \\
\hline PT & 13 & 21 & 15 & 19 & 21 & 21 & 18,3 \\
PSDB & 29 & 31 & 31 & 24 & 34 & 28 & 29,5 \\
PFL/DEM & 33 & 30 & 28 & 28 & 31 & 33 & 30,6 \\
PMDB & 56 & 50 & 57 & 62 & 60 & 47 & 55,3 \\
\hline
\end{tabular}

Fuente: Declaraciones de ingresos y gastos de los directorios nacionales, entregados anualmente al TSE. 
desaparecido por completo -como es el caso de los núcleos de base, uno de los lazos entre partido y sociedad-. En general, tales cambios (lentos y graduales) han aproximado las prácticas del PT a las de sus adversarios: concesión de mayor autonomía a los órganos subnacionales (para las coaliciones electorales, por ejemplo), aflojamiento de los criterios de afiliación, preponderancia de los parlamentarios en el aparato, aproximación del Estado, etc. No obstante, como se defendió en trabajos anteriores (Ribeiro, 2010, 2012), la organización petista todavía constituye, aunque en menor grado, una anomalía en el escenario político brasileño ${ }^{12}$.

Los datos de la Tabla 6 no dejan dudas sobre la validez, en el caso brasileño, de la tendencia de parlamentarización de las cúpulas de los partidos contemporáneos, uno de los síntomas de la cartelización en el plano organizativo (Detterbeck, 2005; Katz y Mair, 2009). La suma entre mandatarios y exmandatarios en cada Ejecutiva Nacional es de al menos $75 \%$ (con un promedio del $85 \%$ ), con el punto máximo en el PMDB. En todos los partidos predominan los diputados federales y senadores y, en segundo lugar, los exocupantes de estos cargos. Los mandatarios subnacionales, como alcaldes y diputados estaduales (pero también gobernadores, que pueden ser líderes nacionales importantes), constituyen la minoría. Considerando que la mayoría de los miembros nunca elegidos eran ocupantes o exocupantes de otros puestos estatales (por medio de nombramientos patrocinados por los partidos), el peso estatal en la profesionalización política de las cúpulas es todavía más significativo.

Tabla 6: Composición de las Comisiones Ejecutivas Nacionales (en \%)

\begin{tabular}{lccccccccc}
\hline & PMDB & PT & PSDB & DEM & PP & PTB & PDT & PR & PSB \\
\hline Mandatarios federales $^{1}$ & 92 & 42 & 45 & 61 & 45 & 34 & 35 & 32 & 27 \\
Mandatarios subnacionales $^{2}$ & - & 11 & 3 & 8 & 5 & 16 & 20 & 18 & 27 \\
Exmandatarios (todos los tipos) & 8 & 32 & 38 & 24 & 40 & 25 & 30 & 27 & 18 \\
$\begin{array}{l}\text { Nunca elegidos para cargos } \\
\text { públicos }\end{array}$ & - & 16 & 14 & 8 & 10 & 25 & 15 & 23 & 27 \\
Total & 100 & 100 & 100 & 100 & 100 & 100 & 100 & 100 & 100 \\
$\mathrm{~N}$ & 13 & 19 & 29 & 38 & 20 & 44 & 20 & 22 & 33 \\
\hline
\end{tabular}

Fuente: las composiciones de las ejecutivas nacionales (vigentes en octubre de 2012) fueron consultadas en los sitios web oficiales de cada partido. Los datos biográficos de cada miembro fueron obtenidos en diversas fuentes, pero principalmente los sitios web de la Cámara de Diputados, Senado Federal y Asambleas Legislativas Estaduales.

${ }^{1}$ Diputados federales y senadores con mandatos vigentes.

${ }^{2}$ Diputados estaduales, gobernadores estaduales, alcaldes y concejales. 


\section{COMPETENCIA Y COOPERACIÓN}

Si la volatilidad ha disminuido en los últimos años, la fragmentación del sistema político brasileño aún es elevada. La cantidad de partidos en la Cámara de Diputados ha aumentado en los últimos comicios, tanto en términos nominales como si calculamos el número efectivo de partidos (Tabla 7). Por otro lado, la estabilización de una dinámica nacional alrededor del PT y PSDB se manifiesta en el cálculo del número efectivo para las elecciones presidenciales, evidenciando las dificultades de inserción de una tercera fuerza relevante en la disputa.

La apreciación de los datos mencionados anteriormente pone de manifiesto una cuestión: ¿cómo un cartel podría establecerse en un sistema tan fragmentado? Ya al principio, no es tan sencillo definir cuáles son los partidos que componen la élite del sistema. El PMDB, PT, PSDB y DEM (a despecho de su decadencia reciente) forman la primera línea; el PP, también heredero (como el DEM) del partido de soporte del régimen militar, sería un quinto elemento imprescindible, considerando su penetración en el territorio y su fuerza electoral en estados y municipios. ${ }^{13}$ Sin embargo, los cinco partidos han obtenido, en conjunto, proporciones declinantes de la Cámara de Diputados: de 80\% de los representantes en 1998, conquistaron menos del $60 \%$ en $2010 .{ }^{14}$ La definición de los demás miembros de la élite se pone frente a dos factores: por un lado, partidos medianos con desempeños electorales oscilantes; por otro, algunos de estos partidos han tenido grados considerables de influencia en la dinámica política nacional, como aliados importantes en las coaliciones de gobierno. Considerando que cualquier criterio es arbitrario y está sujeto a controversias, podríamos tomar como relevantes los partidos que obtuvieron al menos el $4 \%$ de representación en la Cámara de Diputados en las tres

Tabla 7: Fragmentación del sistema político brasileño

\begin{tabular}{lcccccccc}
\hline & 1982 & 1986 & $1989-90$ & 1994 & 1998 & 2002 & 2006 & 2010 \\
\hline $\begin{array}{l}\text { Número efectivo de partidos: } \\
\text { elecciones presidenciales }\end{array}$ & - & - & 5,7 & 2,7 & 2,5 & 3,2 & 2,4 & 2,7 \\
$\begin{array}{l}\text { Número efectivo de partidos } \\
\text { parlamentarios (Cámara) }\end{array}$ & 2,4 & 2,8 & 8,7 & 8,2 & 7,1 & 8,5 & 9,3 & 10,4 \\
$\begin{array}{l}\text { Número de partidos nominales } \\
\text { en la Cámara }\end{array}$ & 5 & 12 & 19 & 18 & 18 & 19 & 21 & 22 \\
\hline
\end{tabular}

Fuente: Melo (2007), actualizado por el autor para 2010, con datos brutos del TSE.

${ }^{1}$ Según la fórmula de Laakso y Taagepera.

13 En los comicios locales de 2012, el PP conquistó el 8,4\% de los alcaldes (más que el DEM), siendo superado por el PMDB (18,3\%), PSDB (12,7\%), PT (11,4\%) y PSD (8,9\%).

14 Los valores fueron el $72 \%$ en 2002 , y el $67 \%$ en 2006 . 
últimas elecciones (2002, 2006 y 2010). Con este criterio, además de los cinco partidos mencionados, pueden ser considerados miembros de la élite el PSB, PDT, PTB y PR. Juntos, los nueve partidos obtuvieron el 91\% de la Cámara en 2002 y el 85\% en 2006 y 2010.

El proceso de creación del PSD ha traído algunas lecciones sobre las posibilidades de cartelización del sistema brasileño. Por un lado, expuso ciertos obstáculos contra el fenómeno. Sin una cláusula de desempeño uniforme, las barreras de entrada en el sistema son reducidas, especialmente en algunos estados del país. Con estructuras y patrones de competencia distintos en cada estado, las oportunidades de entrada y de progreso electoral se abren a casi todos. Además, la regulación sobre el transfuguismo interpartidista era muy floja hasta recientemente, lo que mantenía las puertas abiertas para frecuentes reacomodaciones de la élite política (incluso entre las elecciones), según las conveniencias nacionales o regionales. A partir de 2007, el TSE pasó a considerar que los mandatos pertenecen a los partidos, y no a los políticos individualmente. Sin embargo, el tribunal ha dejado algunas brechas en la regulación, bastante maleables y subjetivas: cambios en el programa del partido, "persecuciones" internas o la creación de un nuevo partido son algunas de las justificaciones que permiten que un político migre de un partido a otro, sin perder el mandato. La última justificación fue suficiente para el PSD, que a mediados de 2012, sin haber disputado ninguna elección, poseía la cuarta bancada en la Cámara, con 50 diputados federales, además de cientos de alcaldes también elegidos por otros partidos. En los comicios locales de octubre del mismo año, el PSD obtuvo 495 ayuntamientos y 4665 concejales municipales, comprobando que el partido ya nacía grande (cuarto mejor desempeño), gracias al apoyo de máquinas y liderazgos estaduales y locales preexistentes, probablemente interesados en acercarse al gobierno federal.

Por otro lado, el episodio ilustró cómo los grandes partidos pueden intentar acciones concertadas para dificultar la entrada de nuevos competidores. Frente a la demanda del PSD por una participación en el fondo partidario y en el tiempo de radio y televisión proporcional al tamaño de su bancada en la Cámara, los partidos que serían los más perjudicados (PMDB, PSDB, PP, PR, PTB y, principalmente, el DEM) articularon una reacción para frenar la demanda. La ausencia de los partidos de izquierda (PT, PSB y PDT) en la articulación posiblemente indicaba un cálculo estratégico: incentivar una escisión que debilitaba principalmente al PSDB y al DEM, opositores al gobierno. Las decisiones del TSE y STF, primero garantizando los mandatos de los parlamentarios del PSD, después el acceso al fondo partidario de manera proporcional al tamaño de su bancada, aseguraron el éxito de la estrategia del partido. Ya dentro del "club", el PSD empezó a articularse con los demás grandes partidos para frenar pretensiones de otros posibles nuevos afiliados, motivados a reproducir la estrategia del propio partido. ${ }^{15}$

Otro caso de cooperación fue la reacción a la decisión del TSE de 2007, que reglamentó la división del fondo partidario y del tiempo de radio y TV. Como la regla transfería

15 "Para deter PSD, 8 siglas fazem guerrilha no Congresso e ação conjunta no TSE". Jornal O Estado de São Paulo, 23 de febrero de 2012. "Dinheiro público estimula criação de novas legendas". Jornal O Estado de São Paulo, 3 de marzo de 2013 
recursos de los partidos más grandes para los demás ${ }^{16}$, dos días después de su publicación, las bancadas de ocho de los nueve partidos principales propusieron un nuevo criterio (vigente hoy), con la división igualitaria del $5 \%$ de los recursos del fondo y el 95\% según la votación para la Cámara de Diputados. El PSB, que no participó en la proposición inicial, y el PDT, que firmó la propuesta pero entró en obstrucción durante la votación, tuvieron un comportamiento ambiguo debido a su participación en un bloque parlamentario recién creado en la Cámara, que contaba con partidos pequeños, beneficiados por la regla del TSE. ${ }^{17}$ El caso demuestra, por un lado, que las estrategias de colusión ni siempre pueden ser explícitas y, por otro, que el poder judicial puede contraponerse a la acción concertada entre los grandes partidos.

La posibilidad de contraposición judicial adquiere una importancia todavía más grande cuando consideramos que la regla vigente hoy, aunque no tan buena a los pequeños partidos como sería el criterio pretendido por el TSE, hizo disminuir la desventaja entre grandes y pequeños en la distribución de los recursos estatales (Gráfico 2). Inversamente a lo que sucedía hasta 2006, los nueve partidos principales empezaron a recibir, en conjunto, una proporción del dinero estatal inferior a la suma de sus bancadas en la Cámara de Diputados. Como otro efecto del nuevo criterio (pero también de la mayor fragmentación de la Cámara), la distancia entre los partidos que componen la élite también ha disminuido (Tabla 8). Para Katz y Mair (2009), este es un síntoma de cartelización, ya que reduce los costos de eventuales derrotas electorales.

Gráfico 2: División del fondo partidario entre los principales partidos y los demás, 1994-2012 (en \%)

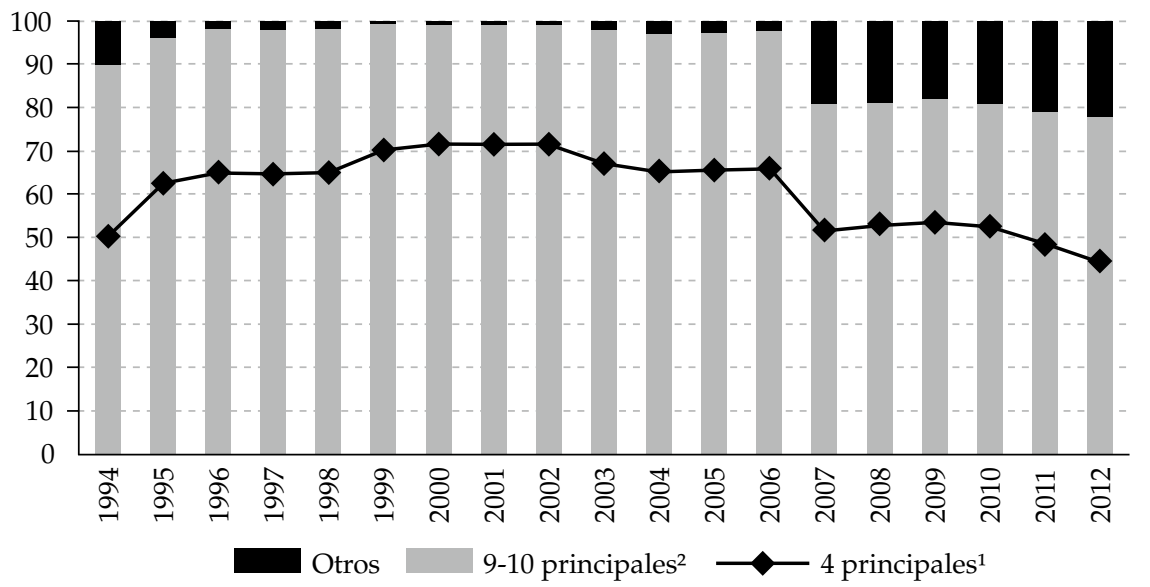

Fuente: calculado a partir de datos oficiales del TSE.

${ }^{1}$ PT, PSDB, PMDB y PFL/DEM.

${ }^{2}$ Incluyen también PP, PTB, PL/PR, PDT y PSB; en 2012, el PSD ha sido incluido.

16 El criterio establecido por el TSE (debido al vacío legal generado tras la declaración, por parte del STF, sobre la inconstitucionalidad de la cláusula de desempeño) determinaba que el $42 \%$ del fondo se dividirían igualitariamente entre todos los partidos y el 58\% según la representación parlamentaria.

17 Las informaciones sobre esta votación y sobre las demás que se presentan adelante fueron extraídas del sitio de la Cámara (www.camara.gov.br) y en los periódicos de la época. 


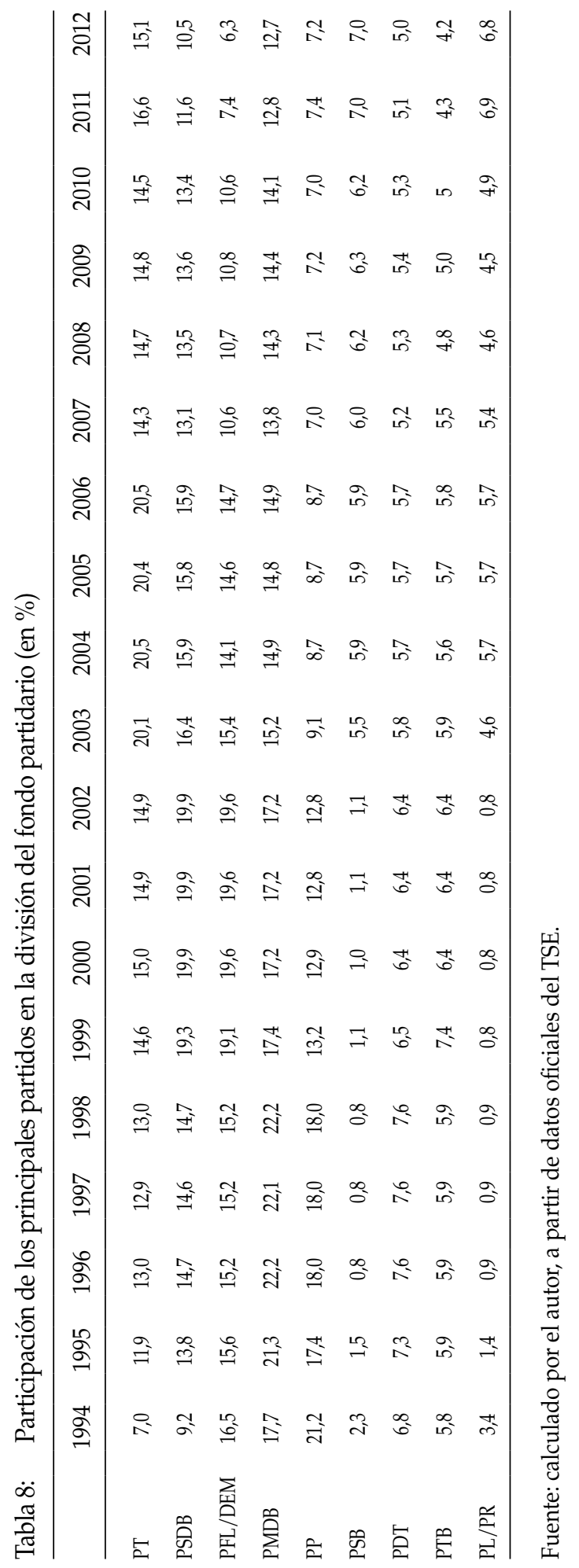


No obstante los casos presentados anteriormente, el análisis de algunas otras cuestiones sensibles no permite el delineamiento de patrones claros y regulares de cooperación entre los grandes partidos. Los líderes de los nueve partidos principales construyeron (en 1998 y 2009) acuerdos respecto de la delimitación de responsabilidad sobre infracciones electorales cometidas por órganos partidistas subnacionales, de manera de no perjudicar a los directorios nacionales en el recibimiento del fondo partidario y también (en 2005) sobre el criterio que determina que los cargos en la Cámara de Diputados (comisiones, mesa directiva, etc.) deben ser distribuidos en proporción a las bancadas partidistas resultantes de la última elección, desconsiderando los cambios de partido hechos por los diputados durante la legislatura. En 1995 se pusieron de acuerdo (incluso el PT) acerca del establecimiento de la cláusula de desempeño del $5 \%$ de los votos para la Cámara y sobre el aplazamiento de la vigencia del dispositivo para los comicios de 2006. Sin embargo, los partidos medianos (principalmente el PL/PR, PTB y PDT), en riesgo de no superar la barrera, intentaron por diversas formas cambiar la regla hasta 2007, cuando el STF declaró su inconstitucionalidad. Divisiones semejantes han ocurrido a partir de 2007 en las discusiones sobre la reforma política: si la propuesta de financiamiento público de campañas recibe un gran apoyo de la clase política, el cambio del sistema electoral muchas veces ha ubicado en campos opuestos los cuatro partidos principales, por un lado, y los partidos medianos, por otro. Tras las manifestaciones callejeras de junio de 2013, la discusión volvió al escenario, y también las disensiones -ahora más acentuadas-. El voto en lista cerrada, la financiación pública de campañas y la convocación de un plebiscito o referéndum sobre la reforma política, algunas de las cuestiones más discutidas, han generado inúmeras divergencias incluso dentro de cada bancada parlamentaria y también entre los grandes partidos. La imposibilidad de formación de consensos mínimos, aunque solo entre los partidos de un eventual cartel, sigue siendo la tónica, dificultando la aprobación de reformas institucionales que favorezcan solamente a las grandes fuerzas.

\section{CONSIDERACIONES FINALES}

La dependencia estatal de los partidos, la hegemonía de la cara pública en las ejecutivas nacionales, la estabilización de la competencia, la disminución de las distancias ideológicas, los indicios de colusión entre los principales partidos (superando los clivajes gobierno/oposición y derecha/izquierda) para la promoción de objetivos comunes, la perspectiva de todos los partidos medianos y grandes en integrar el gobierno y la reducción de la distancia entre ganadores y perdedores, en términos del acceso a los recursos estatales, son algunos factores que sugieren la posibilidad de cartelización de la política brasileña.

Si el modelo del cartel ayuda a iluminar el caso brasileño, el análisis de este caso pone de manifiesto, por otro lado, algunos problemas empíricos y de orden teórico y conceptual del modelo. En primer lugar, la estabilización de la estructura de competencia y la disminución 
de las distancias ideológicas, factores privilegiados en la tesis del cartel, no han traído consigo la reducción de la fragmentación, con sus consecuencias importantes para el sistema de gobierno de Brasil. El PSDB, PT, PMDB y PFL/DEM, que reunían dos tercios de la Cámara en 1999, obtuvieron, en 2010, poco más de la mitad de los diputados. Con la crisis del DEM, en 2013 la participación del grupo había sido reducida a 48\% (o 57\%, si agregamos también el PSD). Con la inviabilidad política de una alianza federal entre PT y PSDB, los partidos medianos, con desempeños oscilando entre el $4 \%$ y el $8 \%$ de los asientos, han sido aliados indispensables de los gobiernos nacionales desde la elección de Fernando Henrique Cardoso en 1994. En el análisis de algunos casos, se identificó que estos partidos medianos han recurrido a su poder de chantaje para defenderse de propuestas que favorecían los cuatro partidos principales, amenazando con represalias en la votación de cuestiones clave para el gobierno de turno. De este modo, estrategias de colusión entre los cuatro grandes, en perjuicio de los demás competidores, tienen pocas posibilidades de éxito. Así, la estabilización de la competencia y la aproximación ideológica no se pueden analizar aisladamente, sin tener en cuenta la fragmentación y los patrones de relación entre ejecutivo y legislativo.

El caso brasileño también pone de manifiesto la fragilidad de las articulaciones empíricas entre financiamiento público, reducción de la competitividad del sistema y cartelización. Al contrario, los datos sugieren -en el mismo sentido planteado por Scarrow (2006)que los recursos públicos quizás estén elevando la competitividad y la fragmentación del sistema al sustentar los partidos pequeños y medianos, que tienen un acceso más difícil a las donaciones de las grandes compañías, destinadas principalmente al PT, PSDB y PMDB. En este sentido, los grandes partidos no han logrado, en los últimos años, frenar las demandas de los demás por parcelas más grandes del fondo partidario y del tiempo de propaganda. Igualmente, no han sido capaces de elevar las barreras de entrada y de acceso a estos recursos. El financiamiento público no ha sido suficiente ni para impedir la aparición de nuevos competidores en Brasil, como el PSD ${ }^{18}$-también beneficiado por la acción del poder judicial y por la continuada ausencia de reglas más rígidas para que los políticos cambien de partido-.

Así, bajo las condiciones actuales, resulta poco factible la formación de un cartel cerrado y estable en Brasil. Un eventual proceso de cartelización solo podría asumir la forma de un "cartel dinámico": cuatro (o cinco, con el PSD) líderes de largo plazo del juego y de cuatro a siete miembros rotatorios, entrando y saliendo del cartel según su desempeño electoral y el contexto político. De cualquier manera, ni siempre sería tan sencillo separar, en términos empíricos, las acciones de cooperación que buscan beneficiar a toda la clase política (como la elevación del total del fondo partidario en 2011) de aquellas que favorecen solamente a los principales partidos. En el primer caso, no se puede hablar de un cartel, sino de corporativismo de la clase política, lo que llevaría la discusión a otros términos (Koole, 1996). 
También hay graves problemas cuando pasamos del plano sistémico al plano organizativo de análisis. En primer lugar, el dominio de los mandatarios es un fenómeno generalizado en las cúpulas de los partidos brasileños, independiente de su posición como miembros o no de un eventual cartel. Además, es un fenómeno general en las democracias contemporáneas (Katz y Mair, 2002). En segundo lugar, los indicios sobre las relaciones internas de poder no apuntan hacia una dirección inequívoca. Las transformaciones en el PSDB y DEM, aunque empezadas anteriormente, fueron aceleradas a partir de la llegada del PT al Palacio do Planalto -que representa un polo magnético muy acentuado, manifestándose de manera drástica, para las oposiciones, en la defección de líderes hacia los partidos gobernantes-. Frente al reto de pasar a la oposición, que generó crisis organizativas serias (Panebianco, 1995), los dirigentes del DEM y del PSDB han intentado (hoy todavía) controlar de modo más centralizado a sus líderes y órganos subnacionales, reforzando la articulación interna para intentar sobrevivir y volver al gobierno. Para el PT, si los cambios efectuados entre 1995 y 2002 tenían como propósito la conquista del gobierno federal, las transformaciones posteriores fueron impulsadas por la necesidad de controlar sectores del partido en momentos críticos (como el comienzo del gobierno Lula) y por la ambición de aumentar su competitividad electoral en estados y municipios, recurriendo a alianzas con fuerzas de todos los campos ideológicos. Así, si unos caminan en dirección a una mayor centralización nacional, la trayectoria del PT es inversa, hacia la ampliación de la autonomía de directorios estaduales y locales (Guarnieri, 2011; Ribeiro, 2013).

Por lo tanto, modelos organizativos extremamente distintos, como son los del PT, PMDB y DEM, pueden coexistir en un sistema con posibilidades de cartelización. La dependencia estatal de los partidos, la hegemonía de la cara pública en las máquinas y las estrategias de cooperación son factores que pueden sostener una relación causal directa, mutuamente reforzadora, sin implicar en la conformación de un modelo organizativo específico. Los retos ambientales (incluso los coyunturales) presionan y motivan a los líderes a adoptar determinadas estrategias organizativas y soluciones adaptativas. Sin embargo, el modelo genético del partido, su desarrollo histórico y sus instituciones internas formales e informales también interfieren en el juego, limitando el margen de maniobra del liderazgo y contribuyendo para el mantenimiento de algunas diferencias organizativas (Panebianco, 1995). Así, una de las inconsistencias teóricas de la tesis del cartel es considerar (aunque implícitamente) que los partidos responderán, en términos organizativos, solamente a los retos más estructurales (como los costos elevados de las campañas o la dilución de los clivajes de clase) y a los supuestos objetivos racionales de sus élites parlamentarias, depreciando otras variables independientes señaladas con anterioridad (tal como las internas), que pueden producir resultados distintos en términos organizativos.

Por lo tanto, la exploración empírica de la dimensión organizativa del modelo y la propia articulación conceptual entre los planos sistémico y organizativo, siguen siendo los principales problemas de la tesis. Cabe señalar aquí que, frente a las críticas, Katz y Mair (2009: 757; 763, nota 4) empezaron a hablar más bien en un "modelo cartel de política". Sin embargo, mantuvieron el carácter inseparable de los planos sistémico y organizativo y la idea del partido cartel como sucesor de los partidos de cuadros, de masas y catch all, el cuarto estadio en la "evolución natural de la especie". 
En este sentido, estamos de acuerdo con Detterbeck (2005): un modelo de política cartelizada (pero no un tipo ideal de partido), vaciado de su componente organizativo y reducido a sus elementos principales, sería más consistente en términos teóricos y más rentable en términos de comparación y comprobación empírica. Un sistema cartelizado podría, así, ser definido como aquel en el que hay algún grado de cooperación entre los partidos principales (a despecho de las fronteras entre gobierno y oposición) para proteger sus posiciones privilegiadas de acceso a los recursos públicos y para mantener o reforzar los importes totales de estos recursos, frente a la situación generalizada de dependencia estatal. Con esta definición mínima tendríamos un modelo más abierto a la multiplicidad de las experiencias democráticas contemporáneas y más apropiado para iluminar el análisis y la comparación de sistemas políticos, enfocando ciertas tendencias generales de transformación, pero sin insistir en los mismos problemas de las antiguas tipologías, como el carácter evolucionista y determinista.

\section{REFERENCIAS}

Alcántara, Manuel. 2004. ¿Instituciones o máquinas ideológicas? Origen, programa y organización de los partidos politicos latinoamericanos. Barcelona: Institut de Ciènces Polítiques i Socials.

Aucante, Yohann. 2008. "L'oligarchie partisane américaine: un modèle de cartel?" En Les systèmes de partis dans les démocraties occidentales: le modèle du parti-cartel en question. Yohann Aucante y Alexandre Dézé (eds.), 297-318. Paris: Sciences Po, le Presses.

Aucante, Yohann y Alexandre Dézé (eds.). 2008. Les systèmes de partis dans les democraties occidentales: Le modèle du parti-cartel en question. Paris: Presses de Sciences Po.

Bardi, Luciano (ed.). 2006. Partiti e sistemi di partito: il cartel party e oltre. Bologna: Il Mulino.

Blyth, Mark y Richard Katz. 2005. "From Catch-all Politics to Cartelisation: the Political Economy of the Cartel Party". West European Politics 28 (1): 33-60.

Detterbeck, Klaus. 2005. “Cartel Parties in Western Europe?”. Party Politics 11 (2): 173-191.

Ferreira, Denise P. 2002. PFL x PMDB: marchas e contramarchas (1982-2000). Goiânia: Alternativa.

Figueiredo, Argelina y Fernando Limongi. 2007. “Instituições políticas e governabilidade: desempenho do governo e apoio legislativo na democracia brasileira". En A democracia brasileira: balanço e perspectivas para 0 século 21. Carlos R. Melo y Manuel Alcántara (eds.), 147-198. Belo Horizonte: UFMG.

Guarnieri, Fernando. 2011. “A Força dos Partidos 'Fracos'”. Dados 54 (1): 235-258.

Heurtaux, Jérôme y Antoine Roger. 2008. "En attendant le cartel... Katz et Mair à l'épreuve des sociétés postcommunistes". En Les systèmes de partis dans les démocraties occidentales: le modèle du parti-cartel en question. Yohann Aucante y Alexandre Dézé (eds.), 275-296. Paris: Presses de Sciences Po.

Hunter, Wendy. 2010. The Transformation of the Workers' Party in Brazil, 1989-2009. New York: Cambridge University Press.

Katz, Richard y Peter Mair. 1995. "Changing Models of Party Organization and Party Democracy: The Emergence of the Cartel Party". Party Politics 1 (1): 5-28.

Katz, Richard y Peter Mair. 1996. "Cadre, Catch-All or Cartel?: A Rejoinder". Party Politics 2 (4): 525-534.

Katz, Richard y Peter Mair. 2002. "The Ascendancy of the Party in Public Office: Party Organizational Change in Twentieth-Century Democracies". En Political Parties: Old Concepts and New Challenges. Richard Gunther, José R. Montero y Juan Linz (eds.), 113-135. Oxford: Oxford University Press.

Katz, Richard y Peter Mair. 2009. “The Cartel Party Thesis: A Restatement”. Perspectives on Politics 7 (4): 753-766.

Kitschelt, Herbert. 2000. "Citizen, Politicians, and Party Cartelization". European Journal of Political Research 37 (2): 149-179.

Koole, Ruud. 1996. "Cadre, Catch-all or Cartel? A Comment on the Notion of the Cartel Party". Party Politics 2 (4): 507-523. 
Kopecký, Petr, Maria Spirova y Gerardo Scherlis. 2011. "Beyond the Cartel Party? Party Patronage and the Nature of Parties in New Democracies". São Paulo, IPSA-ECPR Joint Conference.

Latinobarómetro. 2011. Informe 2011, Santiago de Chile: Latinobarómetro.

Limongi, Fernando y Rafael Cortez. 2010. "As eleições de 2010 e o quadro partidário". Novos Estudos CEBRAP 88.

López Nieto, Lourdes. 2012. Cartelization of the Spanish Political System?. Madrid, XXII World Congress of Political Science (IPSA).

Lucas, Kevin y David Samuels. 2011. "A 'Coerência' ideológica do sistema partidário brasileiro". En O Congresso por ele mesmo: autopercepções da classe política brasileira. Timothy Power y Cesar Zucco (eds.), 61-103. Belo Horizonte: UFMG.

MacIvor, Heather. 1996. "Do Canadian Political Parties form a Cartel?". Canadian Journal of Political Science 29 (2): 317-333.

Melo, Carlos R. 2007. "Nem tanto ao mar, nem tanto a terra: elementos para uma análise do sistema partidário brasileiro". En A democracia brasileira: balanço e perspectivas para o século 21. Carlos R. Melo y Manuel Alcántara (eds.), 267-302. Belo Horizonte: UFMG.

Melo, Carlos R. y Rafael Câmara. 2012. "Estrutura da Competição pela Presidência e Consolidação do Sistema Partidário no Brasil". Dados 55 (1): 71-117.

Nicolau, Jairo. 2010. "Democracy in Brazil, 1985-2006: Moving toward Cartelization". En Political Parties and Democracy - Volume 1: The Americas. Kay Lawson y Jorge Lanzaro (eds.), 101-126. Santa Barbara: Praeger.

Panebianco, Angelo. 1995. Modelos de Partido: organización y poder en los partidos políticos. Madrid: Alianza Editorial.

Power, Timothy y Cesar Zucco (eds.). 2011. O Congresso por ele mesmo: autopercepções da classe política brasileira. Belo Horizonte: UFMG.

Praça, Sérgio, Andréa Freitas y Bruno Hoepers. 2012. "A Rotatividade dos servidores de confiança no governo federal brasileiro". Novos Estudos CEBRAP 94: 91-107.

Ribeiro, Pedro Floriano. 2010. Dos sindicatos ao governo: a organização nacional do PT de 1980 a 2005. São Carlos / São Paulo: EdUFSCar/FAPESP.

Ribeiro, Pedro Floriano. 2012. "Changing for Victory (and Government): Understanding the Transformation of the Workers' Party via an Organizational Approach, 1980-2010". Latin American Centre, Oxford, Workshop The PT from Lula to Dilma: Explaining Change in the Brazilian Worker's Party.

Ribeiro, Pedro Floriano. 2013. "Organização e poder nos partidos brasileiros: Uma análise dos estatutos". Revista Brasileira de Ciência Política 10: 225-265.

Santos, Fabiano. 2000. "Escolhas institucionais e transição por transação: sistemas políticos de Brasil e Espanha em perspectiva comparada". Dados 43 (4).

Santos, Fabiano y Marcio Vilarouca. 2008. "Political Institutions and Governability from FHC to Lula". En Democratic Brazil Revisited. Timothy Power y Peter Kingstone (eds.), 57-80. Pittsburgh: University of Pittsburgh Press.

Scarrow, Susan. 2006. "Party Subsidies and the Freezing of Party Competition: Do Cartel Mechanisms Work?". West European Politics 29 (4): 619-639.

Souza, Maria do Carmo C. 1983. Estado e partidos políticos no Brasil (1930 a 1964). São Paulo: Alfa-Ômega.

Van Biezen, Ingrid y Ekaterina Rashkova. 2011. "Breaking the Cartel: the Effect of State Regulation on New Party Entry". Working Paper Series on the Legal Regulation of Political Parties, n. 12.

Yishai, Yael. 2001. “Bringing Society Back in: Post-cartel Parties in Israel”. Party Politics 7 (6): 667-687.

Pedro Floriano Ribeiro es doctor en Ciencia Política, profesor e investigador del departamento de Ciencias Sociales y del Programa de Postgrado en Ciencia Política de la Universidad Federal de São Carlos (Brasil), donde coordina el Centro de Estudos de Partidos Políticos (CEPP). Se especializa en partidos políticos, organizaciones partidistas y élites políticas. E-mail: pfribeiro@ufscar.br 
